TRANSACTIONS OF THE

AMERICAN MATHEMATICAL SOCIETY

Volume 364, Number 1, January 2012, Pages 103-118

S 0002-9947(2011)05270-0

Article electronically published on August 4, 2011

\title{
POST-CRITICALLY FINITE FRACTAL AND MARTIN BOUNDARY
}

\author{
HONGBING JU, KA-SING LAU, AND XIANG-YANG WANG
}

\begin{abstract}
For an iterated function system (IFS), which we call simple post critically finite, we introduce a Markov chain on the corresponding symbolic space and study its boundary behavior. We carry out some fine estimates of the Martin metric and use them to prove that the Martin boundary can be identified with the invariant set (fractal) of the IFS. This enables us to bring in the boundary theory of Markov chains and the discrete potential theory on this class of fractal sets.
\end{abstract}

\section{INTRODUCTION}

The theory of the Martin boundary is to investigate the limit behavior of the paths of Markov chains. It was introduced by Doob $\mathrm{D}$ and Hunt $\mathrm{H}$ by establishing a probability setting of Martin's work on the positive solution of the Dirichlet problem in an arbitrary domain in $\mathbb{R}^{d}[\mathbf{M}$. The Martin boundary has very rich structure, and the theory has been a cornerstone in probability theory due to its close connection with potential theory and harmonic analysis. Recent development has been associated with random walks on graphs and groups as well as hyperbolic boundaries W.

For a contractive iterated function system (IFS) $\left\{S_{j}\right\}_{j=1}^{N}$ with an invariant set $K$, there is a canonical tree structure on the symbolic space that represents $K$. Recently, Denker and Sato [DS1, DS2] proved that for the special case of Sierpiński gasket, there is a nature transition probability on the tree so that the Martin boundary of the associated Markov chain is homeomorphic to $K$. Moreover, in DS3 they identified a subclass of "strongly harmonic functions" on the Sierpiński gasket that coincides with Kigami's harmonic functions [K1, K2]. The case of the pentagasket and other extensions were studied in [I] and [DIK]. The work of Denker and Sato also initiated some new consideration by Kaimanovich $\mathrm{Ka}$ and the authors [LW] of identifying the self-similar sets as hyperbolic boundaries. It was proved that such identification is possible provided that the generating IFS $\left\{S_{j}\right\}_{j=1}^{N}$ satisfies the open set condition [LW].

The class of post critically finite (p.c.f.) self-similar sets plays a central role in the recent development of analysis on fractals ( $\mathrm{K} 1, \mathbf{K} 2$, , S1, [S2, S3 $)$ : it is a primary class of fractals that is shown to admit Laplacians. They have the properties that

Received by the editors October 12, 2009 and, in revised form, December 4, 2009.

2010 Mathematics Subject Classification. Primary 28A78; Secondary 28A80.

Key words and phrases. Fractals, Green function, harmonic functions, monocyclic, post critically finite, Martin boundary, transition probability.

This research was partially supported by a HKRGC grant and a Direct Grant from CUHK.

(C)2011 American Mathematical Society Reverts to public domain 28 years from publication 
the intersection of $S_{i}(K)$ and $S_{j}(K)$ is a finite set, and the symbolic representations of these points are periodic. Also it was proved that this class of sets satisfies the open set condition in $\mathbb{R}^{2}\left[\mathrm{BR}\right.$, or in $\mathbb{R}^{d}$ if the IFS $\left\{S_{j}\right\}_{j=1}^{N}$ are commensurable [DL].

In this paper we extend the results of [DS1, DS2] to a subclass of "simple" p.c.f. fractals (see Definition 2.1): $S_{i}(K) \cap S_{j}(K)$ is at most one point, and the symbolic representation of the intersection is of the form $i \dot{k}=i k k \cdots$ and $j \dot{\ell}=$ $j \ell \ell \cdots($ monocyclic $)$. A word $\mathbf{u}$ in the symbolic space is used to represent $K_{\mathbf{u}}=$ $S_{u_{1} \cdots u_{n}}(K)$ in the iteration; $\mathbf{u}$ and $\mathbf{v}$ are said to be conjugated if they are either equal, or they are in the same level but with different parents and $K_{\mathbf{u}} \cap K_{\mathbf{v}} \neq \emptyset$. We introduce a Markov chain $\left\{X_{n}\right\}_{n=0}^{\infty}$ on the tree of finite words by assigning equal transition probability from $\mathbf{u}$ to the immediate descendants of $\mathbf{u}$ and its conjugates $\mathbf{v}$ (see $\S 3$ ). Let $\mathcal{M}$ be the Martin boundary with a metric $\rho$ induced by $\left\{X_{n}\right\}_{n=0}^{\infty}$. Our main result is that the Martin boundary $\mathcal{M}$ is homeomorphic to $K$ (Theorem 4.4). Once this is established, then we can identify the space of exits $\mathcal{M}_{\min }$ (minimal harmonic functions) in $K$, and the Poisson-Martin integral representation of positive or bounded harmonic functions will follow.

Unlike DS1, we do not have an explicit expression for the Green function of the Markov chain, we need to adopt a rather different approach in the proofs. For a simple p.c.f. invariant set $K$, the intersections of the iterations induce an equivalence relation $\sim$ on the symbolic space, and the equivalence classes are conjugated words. The equivalence relation has certain distinctive properties (Proposition 2.2). Based on these properties and the given transition probability (see (3.1)), we establish some fine estimates of the Martin metric (Theorem 4.2), which yield the homeomorphism of $K$ and the Martin boundary.

We remark that in $[\mathrm{A}$ (see also $[\mathrm{W}]$ ), Ancona proved that on a hyperbolic graph, the Martin boundary agrees with the hyperbolic boundary under the assumption of a uniformly "irreducible" random walk with bounded range and spectral radius less than 1. As was proved in [LW], there is a large class of self-similar sets that can be identified with hyperbolic boundaries. It is tempting to apply the approach in $\mathrm{A}$ ] to the current setting. However there is a fundamental difference between the two cases: in our consideration the random walk is "one way", whereas in the random walk is reversible and the technique in [A] cannot be applied directly.

We organize the paper as follows. We introduce the class of simple p.c.f. fractals and prove some essential properties on the representing symbolic space in $\S 2$. We set up the Markov chain in $\S 3$. In $\S 4$, we prove the homeomorphism of the Martin boundary with the underlying p.c.f. fractal set. This depends on some estimations of the Martin metric, which we prove in $\S 5$. In $\S 6$, we show that the space of exits equals the Martin boundary, and we obtain the Poisson-Martin integral representation of the positive harmonic functions and bounded harmonic functions.

\section{Preliminaries}

Throughout the paper we assume that $\left\{S_{j}\right\}_{j=1}^{N}$ is an iterated function system (IFS) of injective contractions on $\mathbb{R}^{d}$, i.e., there exists a constant $0<c<1$ such that for each $1 \leq j \leq N$,

$$
0<\left|S_{j}(x)-S_{j}(y)\right| \leq c|x-y| \quad \forall x \neq y \in \mathbb{R}^{d} .
$$


It is well known that there exists a unique nonempty compact subset $K \subset \mathbb{R}^{d}$ such that

$$
K=\bigcup_{j=1}^{N} S_{j}(K)
$$

(see $[\mathrm{F}]$ ). We call $K$ an invariant set of the IFS. In particular, if all the maps are similitudes, we call $K$ a self-similar set.

For an IFS $\left\{S_{j}\right\}_{j=1}^{N}$, we define the associated symbolic spaces and the codings of the points in $K$ as follows. Let $\Sigma=\{1, \ldots, N\}$ be the set of alphabets, let $\Sigma^{*}=\bigcup_{n \geq 0} \Sigma^{n}$ be the set of all finite words $\left(\Sigma^{0}=\{\emptyset\}\right)$, and let $\Sigma^{\infty}=$ $\{1, \ldots, N\}^{\mathbb{N}}$ be the set of infinite words. The length of a word $\mathbf{u}$ is denoted by $|\mathbf{u}|$. For $\mathbf{u}=u_{1} \cdots u_{n}, \mathbf{v}=v_{1} \cdots v_{m} \in \Sigma^{*}$, the concatenation of $\mathbf{u}$ and $\mathbf{v}$ is $\mathbf{u v}=u_{1} \cdots u_{n} v_{1} \cdots v_{m}$. We also use $\mathbf{u}^{k}$ to denote $\mathbf{u}_{1} \mathbf{u}_{2} \cdots \mathbf{u}_{k}$ if $\mathbf{u}_{i}=\mathbf{u}$ for each $i$ $\mathbf{u}^{\infty}$ or $\dot{\mathbf{u}}$ to denote $\mathbf{u u u} \cdots \in \Sigma^{\infty}$, and

$$
\left.\mathbf{u}\right|_{k}= \begin{cases}u_{1} \cdots u_{k} \in \Sigma^{k}, & |\mathbf{u}| \geq k \\ \mathbf{u}, & \text { otherwise }\end{cases}
$$

to denote the truncation of $\mathbf{u}$ (finite or infinite) to length $k$.

For $\mathbf{u}=u_{1} u_{2} \cdots u_{n} \in \Sigma^{*}$, we let $S_{\mathbf{u}}=S_{u_{1}} \circ \cdots \circ S_{u_{n}}$ and $K_{\mathbf{u}}=S_{\mathbf{u}}(K)$ (convention: $S_{\emptyset}=I$ and $\left.K_{\emptyset}=K\right)$. We equip $\Sigma^{\infty}$ with a metric

$$
d(\mathbf{u}, \mathbf{v})=r^{\alpha(\mathbf{u}, \mathbf{v})}
$$

where $0<r<1$ and $\alpha(\mathbf{u}, \mathbf{v})=\max \left\{k:\left.\mathbf{u}\right|_{k}=\left.\mathbf{v}\right|_{k}\right\}$. It follows that $\left(\Sigma^{\infty}, d\right)$ is a compact metric space. For any $\mathbf{u} \in \Sigma^{\infty}$, it is clear that $\bigcap_{k \geq 1} K_{\left.\mathbf{u}\right|_{k}}$ contains only one point. By using this, we can define the projection

$$
\pi: \Sigma^{\infty} \rightarrow K \quad \text { by } \quad\{\pi(\mathbf{u})\}=\bigcap_{k \geq 1} K_{\left.\mathbf{u}\right|_{k}} .
$$

Then $\pi$ is a continuous surjection. For $\mathbf{u}, \mathbf{v} \in \Sigma^{\infty}$, we define

$$
\mathbf{u} \approx \mathbf{v} \quad \text { if and only if } \pi(\mathbf{u})=\pi(\mathbf{v}) .
$$

It is well known that $\tilde{\pi}$ is an equivalence relation. It induces a quotient topology in $\Sigma^{\infty} / \tilde{\pi}$ in the following sense: A subset $B \subset \Sigma^{\infty} / \tilde{\pi}$ is an open set if and only if $\bigcup\{[\mathbf{u}]:[\mathbf{u}] \in B\}$ is an open set in $\Sigma^{\infty}$, where $[\mathbf{u}]$ is the equivalence class of $\mathbf{u}$. It is clear that the quotient map of $\pi$ is a continuous bijection from $\Sigma^{\infty} / \approx$ to $K$, and hence a homeomorphism as the spaces are compact.

Let $\sigma$ be the (left-)shift on $\Sigma^{\infty}$ defined by $\sigma\left(u_{1} u_{2} \cdots\right)=u_{2} u_{3} \cdots$. Following the notation of Kigami [K1, K2], we let

$$
\mathcal{C}_{K}=\bigcup_{i, j \in \Sigma, i \neq j}\left(K_{i} \cap K_{j}\right), \quad \mathcal{C}=\pi^{-1}\left(\mathcal{C}_{K}\right) \quad \text { and } \quad \mathcal{P}=\bigcup_{n \geq 1} \sigma^{n}(\mathcal{C})
$$

$\mathcal{C}$ is called the critical set and $\mathcal{P}$ is called the post critical set. If $\mathcal{P}$ is a finite set, then $\left\{S_{j}\right\}_{j=1}^{N}$ is called a post critically finite (p.c.f.), and $K$ is called a p.c.f. (invariant) set. It is direct to show that for such $K$, every element $\mathbf{u} \in \mathcal{P}$ is eventually periodic.

Definition 2.1. A p.c.f. set $K$ is called monocyclic if each point in $\mathcal{P}$ is of the form $\dot{u}, u \in \Sigma$; furthermore, $K$ is called simple if it is monocyclic and $\#\left(K_{i} \cap K_{j}\right) \leq 1$, for all $i \neq j \in \Sigma$. 
It follows that if $K$ is a simple p.c.f. set, then for $\mathbf{u}, \mathbf{v} \in \Sigma^{\infty}, \pi(\mathbf{u})=\pi(\mathbf{v})$ implies $\mathbf{u}=\mathbf{w} u_{1} \dot{u}_{2}$ and $\mathbf{v}=\mathbf{w} v_{1} \dot{v}_{2}$, where $\mathbf{w} \in \Sigma^{*}$. As in (2.3) the projection $\pi$ induces an equivalence relation $\pi$ on $\Sigma^{\infty}$; it also induces a relation on the set of finite words $\Sigma^{*}$ as follows. Note that for any $\mathbf{u} \in \Sigma^{*}$, we can write $\mathbf{u}=\mathbf{w} u_{1} u_{2}^{k}$ for some $k \geq 1$, we define for $\mathbf{u}, \mathbf{v} \in \Sigma^{*}$

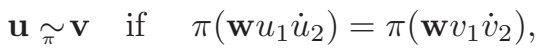

where $\mathbf{u}=\mathbf{w} u_{1} u_{2}^{k}$ and $\mathbf{v}=\mathbf{w} v_{1} v_{2}^{k}$. In particular, $u_{1} u_{2} \approx v_{1} v_{2}$ if and only if $\pi\left(u_{1} \dot{u}_{2}\right)=\pi\left(v_{1} \dot{v}_{2}\right)$. Hence we have

Proposition 2.2. Let $\left\{S_{j}\right\}_{j=1}^{N}$ be a simple p.c.f. IFS. Then $\tilde{\pi}$ defines an equivalence relation on $\Sigma^{*} \cup \Sigma^{\infty}$. Moreover, for $\mathbf{u} \neq \mathbf{v} \in \Sigma^{*} \cup \Sigma^{\infty},|\mathbf{u}|,|\mathbf{v}| \geq 2$, then $\mathbf{u} \approx \mathbf{v}$ if and only if there exists $\mathbf{w} \in \Sigma^{*}, 1 \leq k \leq+\infty$ such that

$$
\mathbf{u}=\mathbf{w} u_{1} u_{2}^{k}, \quad \mathbf{v}=\mathbf{w} v_{1} v_{2}^{k}, \quad \text { and } \quad u_{1} u_{2} \approx v_{1} v_{2}
$$

(here $\left.u_{1} \neq v_{1}\right)$.

We can interpret the geometric meaning $\mathbf{u} \approx \mathbf{v}(|\mathbf{u}|,|\mathbf{v}| \geq 2)$ as either $\mathbf{u}=\mathbf{v}$, or $\mathbf{u}, \mathbf{v}$ have different parents and the intersection of $K_{\mathbf{u}}$ and $K_{\mathbf{v}}$ is a singleton. The relation $\approx$ can be restricted onto $\Sigma^{2}$ and have the following properties:

Theorem 2.3. Let $\left\{S_{j}\right\}_{j=1}^{N}$ be a simple p.c.f. IFS. Then the above relation $\tilde{\pi}$ restricted on $\Sigma^{2}$ is an equivalence relation and satisfies:

(P1) $u_{1} u \approx u_{1} v$ if and only if $u=v$;

(P2) $u u \approx v_{1} v_{2}$ if and only if $u=v_{1}=v_{2}$;

(P3) for any $u_{1} \neq v_{1}$, there exists at most one pair $u_{2}, v_{2}$ such that $u_{1} u_{2} \approx v_{1} v_{2}$.

Proof. To prove $(\mathrm{P} 1)$, let $u_{1} u \approx u_{1} v$, then $\pi\left(u_{1} \dot{u}\right)=\pi\left(u_{1} \dot{v}\right)$. Since $S_{u_{1}}$ is one-toone, it follows that $S_{u}$ and $S_{v}$ have the same fixed point $x_{0}=\pi(\dot{u})=\pi(\dot{v})$. Hence for any $\mathbf{w}=w_{1} \cdots w_{n} \cdots \in \Sigma^{\infty}$, with $w_{n}=u$ or $v$, we have $S_{w_{1} \cdots w_{n}}\left(x_{0}\right)=x_{0}$ for all $n \geq 1$. Let $n \rightarrow \infty$, we have $\pi(\mathbf{w})=x_{0}$ and $\mathbf{w} \in \mathcal{P}$. By the monocyclic property, the only possibility is that $u=v$.

For (P2), if $u u \approx v_{1} v_{2}$, then $\pi(\dot{u})=\pi\left(v_{1} \dot{v}_{2}\right)$, it follows that $\pi(\dot{u})=\pi\left(u^{k} \dot{u}\right)=$ $\pi\left(u^{k} v_{1} \dot{v}_{2}\right)$ for all $k \geq 0$, we see that $u^{k} v_{1} \dot{v}_{2} \in \mathcal{P}$ providing that $u \neq v_{1}$, and this contradicts that $\mathcal{P}$ is a finite set. Hence $u=v_{1}$, and (P1) implies the assertion.

(P3) follows from the assumption that $\#\left(K_{u} \cap K_{v}\right) \leq 1$ for $u \neq v$.

More generally, if we have an equivalence relation $\sim$ on $\Sigma^{2}$ that satisfies $(\mathrm{P} 1)-$ (P3) (not necessarily corresponding to an IFS), then we can extend $\sim$ to $\Sigma^{*} \cup \Sigma^{\infty}$ (as in Proposition 2.2): $\mathbf{u} \sim \mathbf{v}$ if and only if $\mathbf{u}=\mathbf{v}$ or there exist $\mathbf{w} \in \Sigma^{*}$ and $u_{1} u_{2} \sim v_{1} v_{2}$ with $u_{1} \neq v_{1}$ such that

$$
\mathbf{u}=\mathbf{w} u_{1} u_{2}^{k} \text { and } \quad \mathbf{v}=\mathbf{w} v_{1} v_{2}^{k} \quad \text { for some } 0<k \leq \infty .
$$

We have the following converse of Theorem 2.3 when $\sim$ is compatible with $\tilde{\pi}$.

Proposition 2.4. Let $\left\{S_{j}(x)\right\}_{j=1}^{N}$ be an IFS and the equivalence relation $\sim$ on $\Sigma^{2}$ satisfies $(\mathrm{P} 1)-(\mathrm{P} 3)$. Let $\sim$ be extended to $\Sigma^{*} \cup \Sigma^{\infty}$ as (2.5), and suppose that $\mathbf{u}, \mathbf{v} \in \Sigma^{\infty}, \pi(\mathbf{u})=\pi(\mathbf{v})$ implies that $\mathbf{u} \sim \mathbf{v}$. Then the invariant set $K$ is a simple p.c.f. set.

Proof. For any $x \in \mathcal{C}_{K}$, there exist $i, j \in \Sigma(i \neq j)$ and $\mathbf{u}, \mathbf{v} \in \Sigma^{\infty}$ such that $x=\pi(i \mathbf{u})=\pi(j \mathbf{v})$. Therefore, $i \mathbf{u} \approx j \mathbf{v}$. Since $i \neq j$, the assumption on $\sim$ and 
(2.5) imply that $\mathbf{u}=\dot{u}$ and $\mathbf{v}=\dot{v}$ for some $u, v \in \Sigma$ such that $i u \sim j v$. This proves that $K$ is a monocyclic p.c.f. set.

We need to show that $\#\left(K_{i} \cap K_{j}\right) \leq 1$. Suppose otherwise. There exist $i \neq j \in \Sigma$ such that \# $\left(K_{i} \cap K_{j}\right) \geq 2$. Then there exist $\mathbf{u}_{1}, \mathbf{u}_{2}, \mathbf{v}_{1}, \mathbf{v}_{2} \in \Sigma^{\infty}$ such that

$$
\pi\left(i \mathbf{u}_{1}\right)=\pi\left(j \mathbf{v}_{1}\right) \neq \pi\left(i \mathbf{u}_{2}\right)=\pi\left(j \mathbf{v}_{2}\right) .
$$

A similar argument as above shows that there exist $u_{1}, u_{2}, v_{1}, v_{2} \in \Sigma$ such that $\mathbf{u}_{1}=\dot{u}_{1}, \mathbf{u}_{2}=\dot{u}_{2}, \mathbf{v}_{1}=\dot{v}_{1}, \mathbf{v}_{2}=\dot{v}_{2}$. Note that $i u_{1} \sim j v_{1}, i u_{2} \sim j v_{2}$, and by (P3) we have $u_{1}=u_{2}$ and $v_{1}=v_{2}$. This contradicts that $\pi\left(i \mathbf{u}_{1}\right) \neq \pi\left(i \mathbf{u}_{2}\right)$ and the assertion follows.

It is easy to see that the standard polygonal gaskets are simple p.c.f. sets. As an illustration, we use the following "3-level Sierpiński gasket" to explain the equivalence relation $\approx$ on $\Sigma^{2}$.

Example. Let $\left\{p_{1}, p_{2}, p_{3}\right\}$ be the vertices of an equilateral triangle in $\mathbb{R}^{2}$. Let $q_{i}$ be as in Figure 1 with $p_{1}=q_{1}$, and let

$$
S_{j}(x)=\frac{x}{3}+q_{j}, \quad j=1, \ldots, 6 .
$$

The IFS $\left\{S_{j}\right\}_{j=1}^{6}$ generates the 3-level Sierpiński gasket $K$. It is clear that $\tilde{\pi}$ on $\Sigma^{2}$ satisfies $i u \approx j v$ if $K_{i} \cap K_{j} \neq \emptyset($ at $\pi(i \dot{u})=\pi(j \dot{v}))$ (see Figure 1). The words $\mathbf{u}$ will represent the $K_{\mathbf{u}}$ in the gasket. Obviously, the equivalence relation $\approx$ satisfies (P1)-(P3). There are four kinds of equivalent classes, namely, $[11]=\{11\},[54]=$ $\{54\},[13]=\{13,21\},[43]=\{43,25,61\}$.
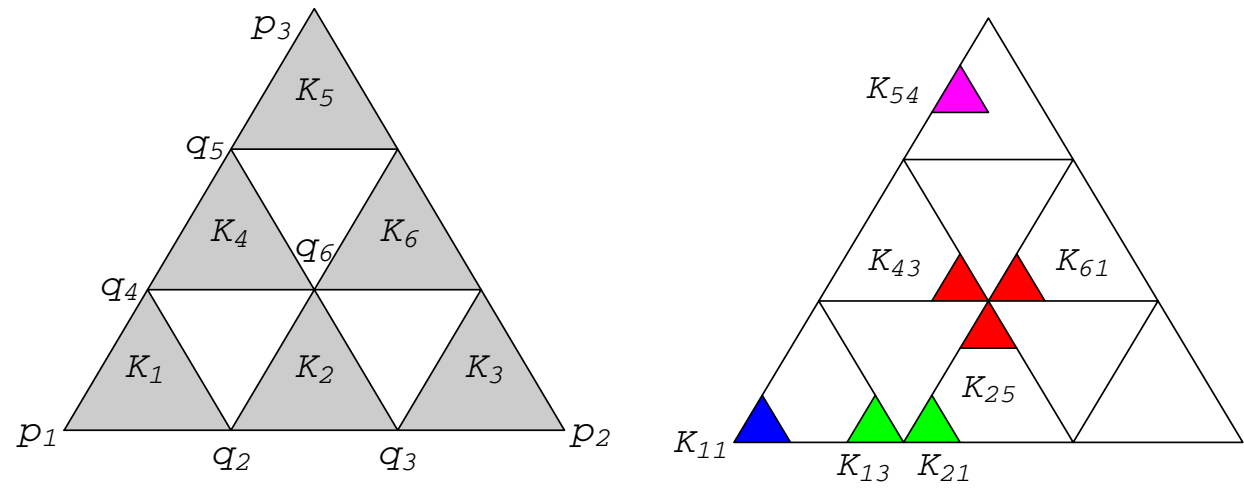

Figure 1

To conclude this section, we remark that it is not clear whether $K$ being monocyclic will suffice for $K$ to be simple. However, we can prove this is true in the following special case.

Proposition 2.5. Let $S_{j}(x)=A\left(x-d_{j}\right)+d_{j}$, where $A$ is a $d \times d$ nonsingular contractive matrix, $d_{j} \in \mathbb{R}^{d}, j=1, \ldots, N$. Suppose the invariant set (self-affine set) $K$ is monocyclic. Then it is simple. 
Proof. Suppose otherwise. There exist $i \neq j \in \Sigma$ such that $\#\left(K_{i} \cap K_{j}\right) \geq 2$, hence there exist $x \neq y \in K_{i} \cap K_{j} \subset \mathcal{C}_{K}$. Since $K$ is monocyclic, we can write

$$
x=\pi\left(i u_{1}\right)=\pi\left(j \dot{u}_{2}\right) \quad \text { and } \quad y=\pi\left(i \dot{v}_{1}\right)=\pi\left(j \dot{v}_{2}\right)
$$

for some $u_{1}, u_{2}, v_{1}, v_{2} \in \Sigma$.

For $\mathbf{u} \in \Sigma^{*}$ with $|\mathbf{u}|>0$, let $e_{\mathbf{u}}=\pi(\dot{\mathbf{u}})$. It is easy to show that $e_{\mathbf{u}}$ is the fixed point of $S_{\mathbf{u}}$ on $\mathbb{R}^{d}$, and we can write $S_{\mathbf{u}}(x)$ as

$$
S_{\mathbf{u}}(x)=A^{|\mathbf{u}|}\left(x-e_{\mathbf{u}}\right)+e_{\mathbf{u}} .
$$

Using this, we have

$$
\pi\left(i u_{1}^{n} \dot{v}_{1}\right)=S_{i}\left(S_{u_{1}^{n}}\left(e_{v_{1}}\right)\right)=A^{n+1}\left(e_{v_{1}}-e_{u_{1}}\right)+A\left(e_{u_{1}}-d_{i}\right)+d_{i} .
$$

By (2.6) and (2.8), it is direct to check that

$$
\pi\left(i u_{1}^{n} \dot{v}_{1}\right)=\pi\left(j u_{2}^{n} \dot{v}_{2}\right), \quad \forall n>0 .
$$

It follows that $u_{1}^{n} \dot{v}_{1}, u_{2}^{n} \dot{v}_{2} \in \mathcal{P}$ for all $n>0$, and hence $\mathcal{P}$ is an infinite set. This is a contradiction and completes the proof.

\section{The transition PROBABILITY}

In the rest of the paper our basic set up is the equivalence relation $\sim$ on $\Sigma^{2}$ that satisfies (P1)-(P3) (as in Theorem 2.3, but it is not necessary to refer to any IFS unless otherwise specified):

(P1) $u_{1} u \sim u_{1} v$ if and only if $u=v$;

(P2) $u u \sim v_{1} v_{2}$ if and only if $u=v_{1}=v_{2}$;

(P3) for any $u_{1} \neq v_{1}$, there exists at most one pair $u_{2}, v_{2}$ such that $u_{1} u_{2} \sim v_{1} v_{2}$. We call $\sim$ the conjugate relation. It extends to an equivalence relation on $\Sigma^{*}$ and $\Sigma^{\infty}$. In view of Theorem 2.3 and Proposition 2.4, a geometric realization of the relation is the simple p.c.f. invariant set.

For $\mathbf{u} \in \Sigma^{*}$ (or in $\Sigma^{\infty}$ ), we let $[\mathbf{u}]$ denote the equivalent class of $\sim$, and we say that elements in $[\mathbf{u}]$ are conjugated. First we introduce a Markov chain $\left\{X_{n}\right\}_{n=0}^{\infty}$ on $\Sigma^{*}$ with a transition probability $p(\cdot, \cdot)$.

Definition 3.1. The transition probability on the state space $\Sigma^{*}$ is defined as

$$
p(\mathbf{v}, \mathbf{w})=\left\{\begin{array}{cr}
\frac{1}{\sharp[\mathbf{v}] N}, & \text { if } \mathbf{w}^{-} \sim \mathbf{v}, \\
0, & \text { otherwise }
\end{array}\right.
$$

where $\mathbf{w}^{-}$is obtained by deleting the last letter of $\mathbf{w}$.

The realization of this transition probability is that if the chain is at state $\mathbf{v}$, then in the next step, it will move to the states $\mathbf{u} j$, the descendants of the conjugate(s) $\mathbf{u}$ of $\mathbf{v}$, with equal probability. Figure 2 is an illustration of the conjugate words and the transition probability corresponding to the Sierpinski gasket DS1. Each word $\mathbf{u}$ is used to represent $K_{\mathbf{u}}$; those words connected by solid line segments are conjugated. For example, $\mathbf{u}=21$ and $\mathbf{v}=12$ are conjugated as $K_{1}$ and $K_{2}$ intersect at $\pi(1 \dot{2})=\pi(2 \dot{1})$; the chain at $\mathbf{u}$ will move to the six descendants of $\mathbf{u}$ and $\mathbf{v}$ with probability $1 / 6$; on the other hand the chain starting from 11 will move to its three descendants with probability $1 / 3$. In the sequel, there are some rather tedious combinatorial arguments and the reader is advised to refer to the Sierpiński gasket or the Example above for a clearer geometric picture in the steps of the proofs. 


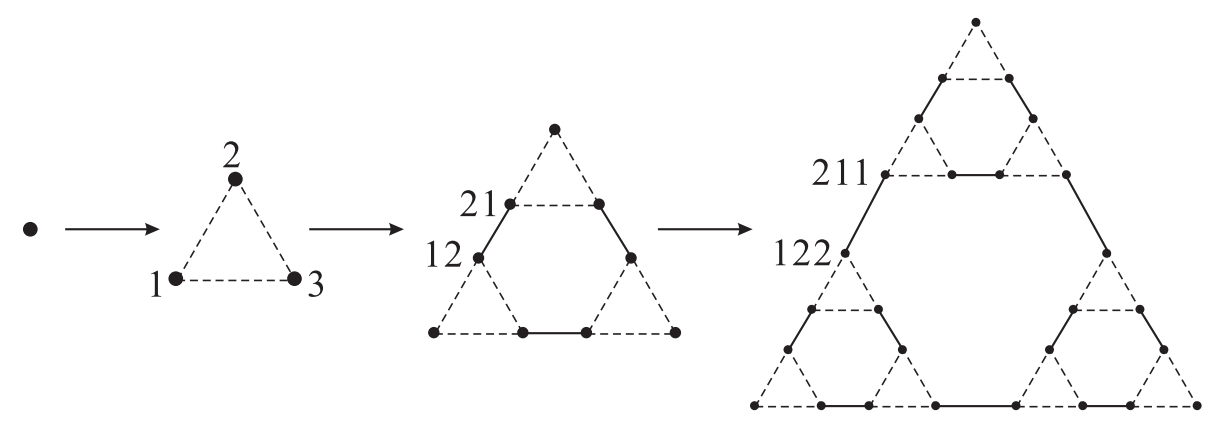

FiguRE 2

Let $p_{n}(\cdot, \cdot)$ be the $n$-step transition probability. It follows from Definition 3.1 that $p_{n}(\mathbf{v}, \mathbf{w})>0$ only if $|\mathbf{w}|-|\mathbf{v}|=n$. A word $\mathbf{v}$ is called an ancestor of $\mathbf{w}$ (also $\mathbf{w}$ is called a successor of $\mathbf{v})$ if $p_{n}(\mathbf{v}, \mathbf{w})>0$ for some $n>0$. We denote this by $\mathbf{v} \prec \mathbf{w}$ and call $\mathbf{v}$ an $n$-th ancestor of $\mathbf{w}$ if we need to specify the generation. Let

$$
\mathcal{A}_{n}(\mathbf{w})=\left\{\mathbf{v} \in \Sigma^{*}: p_{n}(\mathbf{v}, \mathbf{w})>0\right\}
$$

denote the set of $n$-th ancestors of $\mathbf{w}$. It is easy to show from (3.1) that for $\mathbf{w}_{n}=\mathbf{u} w_{0} w_{1} \cdots w_{n}$,

$$
\left[\mathbf{w}_{0}\right] \subset \mathcal{A}_{n}\left(\mathbf{w}_{n}\right) \text { and } \mathcal{A}_{1}\left(\mathbf{w}_{n}\right)=\left[\mathbf{w}_{n}^{-}\right]=\left[\mathbf{w}_{n-1}\right] .
$$

The following proposition is a direct consequence of the definition and induction.

Proposition 3.2. For $\mathbf{w} \in \Sigma^{*}$ with $|\mathbf{w}|=\ell$, then

(i) $p_{\ell}(\emptyset, \mathbf{w})=N^{-\ell}$;

(ii) for $\mathbf{v}_{1} \sim \mathbf{v}_{2}, p_{n}\left(\mathbf{v}_{1}, \mathbf{w}\right)=p_{n}\left(\mathbf{v}_{2}, \mathbf{w}\right)$ for any $n>0$;

(iii) for $\mathbf{v}^{-} \sim \mathbf{w}^{-}, p_{n}(\mathbf{u}, \mathbf{v})=p_{n}(\mathbf{u}, \mathbf{w})$ for any $\mathbf{u} \in \Sigma^{*}, n>0$.

Corollary 3.3. Let $\mathbf{w} \in \Sigma^{*}, n \geq 1$. Then $\sum_{\mathbf{u} \in \mathcal{A}_{n}(\mathbf{w})} p_{n}(\mathbf{u}, \mathbf{w})=N^{-n}$.

Proof. Let $\ell=|\mathbf{w}|$. Then by Proposition $3.2(\mathrm{i})$, we have

$$
N^{-\ell}=p_{\ell}(\emptyset, \mathbf{w})=\sum_{\mathbf{u} \in \mathcal{A}_{n}(\mathbf{w})} p_{\ell-n}(\emptyset, \mathbf{u}) p_{n}(\mathbf{u}, \mathbf{w})=N^{-(\ell-n)} \sum_{\mathbf{u} \in \mathcal{A}_{n}(\mathbf{w})} p_{n}(\mathbf{u}, \mathbf{w}) .
$$

From Proposition 3.2(iii) the next corollary follows.

Corollary 3.4. Assume that $a b \sim c d, a, b, c, d \in \Sigma$ and $k>1$, then

$$
p_{n}\left(\mathbf{v}, \mathbf{w} a b^{k}\right)=p_{n}\left(\mathbf{v}, \mathbf{w} c d^{k}\right), \quad \forall \mathbf{v}, \mathbf{w} \in \Sigma^{*}, n>0 .
$$

We remark that Corollary 3.4 does not hold for $k=1$. Indeed, we see that $p(\mathbf{v} a, \mathbf{v} a b) \geq N^{-2}$, but $p(\mathbf{v} a, \mathbf{v} c d)=0$ if $a \neq c$. We have the following estimation in regard to $\mathbf{w} a b$ and $\mathbf{w} c d$ which will be needed in Proposition 3.6.

Lemma 3.5. Let $\mathbf{w}_{n}=\mathbf{u} w_{0} w_{1} \cdots w_{n}, n \geq 2$. Then for any $\mathbf{v} \in \Sigma^{*}, a, b, c, d \in \Sigma$,

$$
\left|p_{n}\left(\mathbf{v}, \mathbf{w}_{n-2} a b\right)-p_{n}\left(\mathbf{v}, \mathbf{w}_{n-2} c d\right)\right| \leq \gamma^{n-2} N^{-n},
$$

where $\gamma=1-N^{-2}$. 
Proof. We prove this lemma by induction. For $n=2$, by Corollary 3.3 , we have that $0 \leq p_{2}\left(\mathbf{v}, \mathbf{w}_{0} a b\right), p_{2}\left(\mathbf{v}, \mathbf{w}_{0} c d\right) \leq N^{-2}$, and (3.4) follows trivially. For the induction step, observe that

$$
\begin{aligned}
& \left|p_{n+1}\left(\mathbf{v}, \mathbf{w}_{n-1} a b\right)-p_{n+1}\left(\mathbf{v}, \mathbf{w}_{n-1} c d\right)\right| \\
= & \left|\frac{1}{\#\left[\mathbf{w}_{n-1} a\right] N} \sum_{\boldsymbol{\tau} \in\left[\mathbf{w}_{n-1} a\right]} p_{n}(\mathbf{v}, \boldsymbol{\tau})-\frac{1}{\#\left[\mathbf{w}_{n-1} c\right] N} \sum_{\boldsymbol{\eta} \in\left[\mathbf{w}_{n-1} c\right]} p_{n}(\mathbf{v}, \boldsymbol{\eta})\right| \\
= & \frac{1}{\#\left[\mathbf{w}_{n-1} a\right] \#\left[\mathbf{w}_{n-1} c\right] N}\left|\sum_{\boldsymbol{\tau} \in\left[\mathbf{w}_{n-1} a\right]} \sum_{\boldsymbol{\eta} \in\left[\mathbf{w}_{n-1} c\right]}\left(p_{n}(\mathbf{v}, \boldsymbol{\tau})-p_{n}(\mathbf{v}, \boldsymbol{\eta})\right)\right| .
\end{aligned}
$$

Since $p_{n}\left(\mathbf{v}, \mathbf{w}_{n-1} a\right)=p_{n}\left(\mathbf{v}, \mathbf{w}_{n-1} c\right)$, there are at most $\#\left[\mathbf{w}_{n-1} a\right] \#\left[\mathbf{w}_{n-1} c\right]-1$ nonzero terms in the summation. We need only prove that

$$
\left|p_{n}(\mathbf{v}, \boldsymbol{\tau})-p_{n}(\mathbf{v}, \boldsymbol{\eta})\right| \leq \gamma^{n-2} N^{-n} .
$$

Then the induction step follows from

$$
\frac{\#\left[\mathbf{w}_{n-1} a\right] \#\left[\mathbf{w}_{n-1} c\right]-1}{\#\left[\mathbf{w}_{n-1} a\right] \#\left[\mathbf{w}_{n-1} c\right]} \leq 1-N^{-2}=\gamma .
$$

To this end, we need to check that the induction hypothesis can be applied to (3.5). Observe that for $\boldsymbol{\tau} \in\left[\mathbf{w}_{n-1} a\right]$, the conjugation implies that $\boldsymbol{\tau}=\mathbf{w}_{n-2}$ ij if $a \neq w_{n-1}$; on the other hand if $a=w_{n-1}$, then necessarily $\boldsymbol{\tau} \in\left[\mathbf{w}_{n-2} w_{n-1}^{2}\right]$ (by (P2)) and by Corollary 3.4 $p_{n}(\mathbf{v}, \boldsymbol{\tau})=p_{n}\left(\mathbf{v}, \mathbf{w}_{n-2} w_{n-1}^{2}\right)$. The same situation holds for $\boldsymbol{\eta} \in\left[\mathbf{w}_{n-1} c\right]$. Therefore, we can apply the induction hypothesis and complete the proof.

Our main result in this section is the following proposition which is needed to consider the Martin boundary.

Proposition 3.6. Let $\mathbf{w}_{n}=\mathbf{u} w_{0} w_{1} \cdots w_{n}$. Then for $\gamma=1-N^{-2}$,

$$
\left|N^{n} p_{n}\left(\mathbf{v}, \mathbf{w}_{n}\right)-N^{m} p_{m}\left(\mathbf{v}, \mathbf{w}_{m}\right)\right| \leq \frac{\gamma^{m-1}}{1-\gamma} \quad \text { for } n \geq m \geq 1,
$$

and $\left\{N^{n} p_{n}\left(\mathbf{v}, \mathbf{w}_{n}\right)\right\}_{n=1}^{\infty}$ is a Cauchy sequence.

Proof. We need only show that

$$
\left|N^{n+1} p_{n+1}\left(\mathbf{v}, \mathbf{w}_{n+1}\right)-N^{n} p_{n}\left(\mathbf{v}, \mathbf{w}_{n}\right)\right| \leq \gamma^{n-1}
$$

and the proposition follows readily from this. It is easy to check the case $n=1$. For the induction step $n+1$, we consider two separate cases.

Case (i). $w_{n}=w_{n-1}$. Then

$$
\begin{aligned}
p_{n+1}\left(\mathbf{v}, \mathbf{w}_{n+1}\right) & =\sum_{\mathbf{u} \in\left[\mathbf{w}_{n-2} w_{n-1}^{2}\right]} p_{n}(\mathbf{v}, \mathbf{u}) \cdot p\left(\mathbf{u}, \mathbf{w}_{n+1}\right) \\
& =\sum_{\mathbf{u} \in\left[\mathbf{w}_{n-2} w_{n-1}^{2}\right]} \frac{p_{n}\left(\mathbf{v}, \mathbf{w}_{n}\right)}{\#\left[\mathbf{w}_{n-2} w_{n-1}^{2}\right] \cdot N} \quad \text { (by Corollary 3.4) } \\
& =N^{-1} p_{n}\left(\mathbf{v}, \mathbf{w}_{n}\right) .
\end{aligned}
$$

This implies $N^{n+1} p_{n+1}\left(\mathbf{v}, \mathbf{w}_{n+1}\right)=N^{n} p_{n}\left(\mathbf{v}, \mathbf{w}_{n}\right)$ and induction follows. 
Case (ii). $w_{n} \neq w_{n-1}$. Observe that for $\boldsymbol{\tau} \in\left[\mathbf{w}_{n}\right] \backslash\left\{\mathbf{w}_{n}\right\}$, then $\boldsymbol{\tau}=\mathbf{w}_{n-2} c d$ where $c d \sim w_{n-1} w_{n}$. We have

$$
\begin{aligned}
& \left|N^{n+1} p_{n+1}\left(\mathbf{v}, \mathbf{w}_{n+1}\right)-N^{n} p_{n}\left(\mathbf{v}, \mathbf{w}_{n}\right)\right| \\
= & \left|\left(\frac{1}{\#\left[\mathbf{w}_{n}\right]} \sum_{\boldsymbol{\tau} \in\left[\mathbf{w}_{n}\right]} N^{n} p_{n}(\mathbf{v}, \boldsymbol{\tau})\right)-N^{n} p_{n}\left(\mathbf{v}, \mathbf{w}_{n}\right)\right| \\
\leq & \frac{1}{\#\left[\mathbf{w}_{n}\right]} \sum_{\boldsymbol{\tau} \in\left[\mathbf{w}_{n}\right]}\left|N^{n} p_{n}(\mathbf{v}, \boldsymbol{\tau})-N^{n} p_{n}\left(\mathbf{v}, \mathbf{w}_{n}\right)\right| \\
= & \frac{1}{\#\left[\mathbf{w}_{n}\right]} \sum_{\boldsymbol{\tau} \in\left[\mathbf{w}_{n}\right] \backslash\left\{\mathbf{w}_{n}\right\}}\left|N^{n} p_{n}(\mathbf{v}, \boldsymbol{\tau})-N^{n} p_{n}\left(\mathbf{v}, \mathbf{w}_{n}\right)\right| \\
\leq & \frac{\#\left[\mathbf{w}_{n}\right]-1}{\#\left[\mathbf{w}_{n}\right]} \cdot \gamma^{n-2} \quad(\text { by Lemma 3.5]) } \\
\leq & \gamma^{n-1} .
\end{aligned}
$$

\section{MARTin BOUNDARY}

Following the standard notation, we define the Green function and the Martin kernel $g, k: \Sigma^{*} \times \Sigma^{*} \rightarrow \mathbb{R}_{+}$by

$$
g(\mathbf{v}, \mathbf{w})=\sum_{n=0}^{\infty} p_{n}(\mathbf{v}, \mathbf{w}) \quad \text { and } \quad k(\mathbf{v}, \mathbf{w})=\frac{g(\mathbf{v}, \mathbf{w})}{g(\emptyset, \mathbf{w})} .
$$

It follows from (3.1) and Proposition 3.2(i) that $g(\mathbf{v}, \mathbf{w})=p_{|\mathbf{w}|-|\mathbf{v}|}(\mathbf{v}, \mathbf{w})$ and

$$
k(\mathbf{v}, \mathbf{w})=N^{|\mathbf{w}|} g(\mathbf{v}, \mathbf{w}) \leq N^{|\mathbf{v}|}, \quad \mathbf{v}, \mathbf{w} \in \Sigma^{*} .
$$

We define the Martin metric on $\Sigma^{*}$ by

$$
\rho(\mathbf{u}, \mathbf{w})=\sum_{n=0}^{\infty}\left(\frac{r}{N}\right)^{n} \sup _{\boldsymbol{\tau} \in \Sigma^{n}}|k(\boldsymbol{\tau}, \mathbf{u})-k(\boldsymbol{\tau}, \mathbf{w})|,
$$

where $0<r<\gamma\left(=1-N^{-2}\right)$. We use $\sim$ to denote the equivalence relation of $\rho$-Cauchy sequences, and let $\llbracket\left\{\mathbf{w}_{n}\right\} \rrbracket$ denote the equivalence class of a $\rho$-Cauchy sequence $\left\{\mathbf{w}_{n}\right\}$ in $\Sigma^{*}$. If $\left|\mathbf{w}_{n}\right| \rightarrow \infty$ and $\left|\mathbf{v}_{n}\right| \rightarrow \infty$, by the definition of $\rho(\cdot, \cdot)$, it is clear that $\left\{\mathbf{w}_{n}\right\} \widetilde{\rho}\left\{\mathbf{v}_{n}\right\}$ if and only if

$$
k\left(\boldsymbol{\tau}, \mathbf{w}_{n}\right)-k\left(\boldsymbol{\tau}, \mathbf{v}_{n}\right) \longrightarrow 0 \text { for any } \boldsymbol{\tau} \in \Sigma^{*} .
$$

Let $\bar{\Sigma}^{*}$ be the collection of all equivalence classes of $\rho$-Cauchy sequences in $\Sigma^{*}$. Then $\bar{\Sigma}^{*}$ is the $\rho$-completion of $\Sigma^{*}$ and is called the Martin space; it is a compact metric space (we still use $\rho$ to denote the extension) and $\Sigma^{*}$ is an open dense subset of $\bar{\Sigma}^{*}$. The set $\mathcal{M}=\partial \bar{\Sigma}^{*}=\overline{\Sigma^{*}} \backslash \Sigma^{*}$ is called the Martin boundary. Clearly, it is also a compact metric space.

Let $\mathbf{w}=w_{1} w_{2} \cdots \in \Sigma^{\infty}$. Proposition 3.6 implies that for $\left.\mathbf{w}\right|_{n}=w_{1} \cdots w_{n}$ and for any $\mathbf{v} \in \Sigma^{*}$, the sequence $\left\{k\left(\mathbf{v},\left.\mathbf{w}\right|_{n}\right)\right\}$ is a Cauchy sequence. Hence $\lim _{n \rightarrow \infty} k\left(\mathbf{v},\left.\mathbf{w}\right|_{n}\right)$ exists, equivalently, the sequence $\left\{\left.\mathbf{w}\right|_{n}\right\}$ is a $\rho$-Cauchy sequence in $\Sigma^{*}$. We can regard $\left\{\left.\mathbf{w}\right|_{n}\right\}$ as an element in the Martin boundary $\mathcal{M}=\partial \bar{\Sigma}^{*}$. We also extend $\rho$ to $\Sigma^{\infty}$ by

$$
\rho(\mathbf{v}, \mathbf{w})=\lim _{n \rightarrow \infty} \rho\left(\left.\mathbf{v}\right|_{n},\left.\mathbf{w}\right|_{n}\right), \quad \mathbf{u}, \mathbf{v} \in \mathbf{\Sigma}^{\infty} .
$$


Lemma 4.1. Let $\left\{\mathbf{w}_{n}\right\}$ be any $\rho$-Cauchy sequence in $\Sigma^{*}$ with $\left|\mathbf{w}_{n}\right| \rightarrow \infty$ as $n \rightarrow \infty$, and let $\mathbf{w}_{n}^{*} \sim \mathbf{w}_{n}$, where $\sim$ is the conjugate relation defined in the last section. Then $\left\{\mathbf{w}_{n}^{*}\right\}$ is also a $\rho$-Cauchy sequence and $\left.\left\{\mathbf{w}_{n}^{*}\right\} \sim \mathbf{w}_{n}\right\}$. In particular, for $\mathbf{u}, \mathbf{v} \in \Sigma^{\infty}$ with $\mathbf{u} \sim \mathbf{v}$, we have $\left\{\left.\mathbf{u}\right|_{n}\right\} \sim\left\{\left.\mathbf{v}\right|_{n}\right\}$.

Proof. For any $\boldsymbol{\tau} \in \Sigma^{*}$, Corollary 3.4 and Lemma 3.5 imply

$$
\left|k\left(\boldsymbol{\tau}, \mathbf{w}_{n}\right)-k\left(\boldsymbol{\tau}, \mathbf{w}_{n}^{*}\right)\right| \leq N^{|\boldsymbol{\tau}|} \gamma^{\left|\mathbf{w}_{n}\right|-|\boldsymbol{\tau}|-2} \rightarrow 0 \quad \text { as } n \rightarrow \infty .
$$

The first part of the assertion follows from this and (4.3).

For the second part, we let $\mathbf{u}, \mathbf{v} \in \Sigma^{\infty}$ and $\mathbf{u} \sim \mathbf{v}$, we claim that $\left.\left.\mathbf{u}\right|_{n} \sim \mathbf{v}\right|_{n}$ for all but at most one $n$. Indeed it is trivial if $\mathbf{u}=\mathbf{v}$; otherwise $\mathbf{u}$ and $\mathbf{v}$ have the representation $\mathbf{u}=\boldsymbol{\tau} a \dot{b}, \mathbf{v}=\boldsymbol{\tau} c \dot{d}$ with $a b \sim c d, a \neq c$, then $\left.\left.\mathbf{u}\right|_{n} \sim \mathbf{v}\right|_{n}$ except possibly at $n=|\boldsymbol{\tau}|+1$ as claimed. Now by the first part, we have $\left\{\left.\mathbf{u}\right|_{n}\right\} \sim\left\{\left.\mathbf{v}\right|_{n}\right\}$.

The converse of the last statement of Lemma 4.1 is also true, but it is not so direct. It will follow from the following theorem, which is a much stronger statement.

Recall that on $\Sigma^{\infty}$, we have a canonical metric defined by $d(\mathbf{u}, \mathbf{v})=\gamma^{\alpha(\mathbf{u}, \mathbf{v})}$, where $\alpha(\mathbf{u}, \mathbf{v})=\max \left\{k:\left.\mathbf{u}\right|_{k}=\left.\mathbf{v}\right|_{k}\right\}$. For the Martin boundary, we let

$$
\beta(\mathbf{u}, \mathbf{v})=\max \left\{k:\left.\left.\mathbf{u}\right|_{k} \sim \mathbf{v}\right|_{k}\right\}, \quad \mathbf{u}, \mathbf{v} \in \Sigma^{\infty} .
$$

Theorem 4.2. For $\gamma=1-N^{-2}$ and for $0<r<\gamma$, there exist $C_{1}, C_{2}>0$ such that

$$
C_{1} r^{\beta(\mathbf{u}, \mathbf{v})} \leq \rho(\mathbf{u}, \mathbf{v}) \leq C_{2} \gamma^{\beta(\mathbf{u}, \mathbf{v})}
$$

for all $\mathbf{u}, \mathbf{v} \in \Sigma^{\infty}$.

Since the proof requires a few technical lemmas, we will postpone the details of this theorem to the next section. In the following we make use of Theorem 4.2 to conclude the major results of the paper. First we know that $\rho$ is extended naturally to be a metric on the Martin boundary $\mathcal{M}=\partial \bar{\Sigma}^{*}$ in the abstract setting. As a consequence of Lemma 4.1 and Theorem 4.2, we have

Theorem 4.3. The Martin metric $\rho$ on $\Sigma^{*}$ induces a metric (also denote by $\rho$ ) on $\Sigma^{\infty} / \sim$, and the Martin boundary $(\mathcal{M}, \rho)$ is isometrically homeomorphic to $\left(\Sigma^{\infty} / \sim, \rho\right)$.

Proof. Note that $\rho$ is a metric on $\Sigma^{*}$. For any $\mathbf{u} \sim \mathbf{u}^{\prime}, \mathbf{v} \sim \mathbf{v}^{\prime}$ in $\Sigma^{\infty}$, Lemma 4.1 implies that

$$
\begin{aligned}
\rho(\mathbf{u}, \mathbf{v}) & =\lim _{n \rightarrow \infty} \rho\left(\left.\mathbf{u}\right|_{n},\left.\mathbf{v}\right|_{n}\right) \\
& \leq \lim _{n \rightarrow \infty}\left(\rho\left(\left.\mathbf{u}\right|_{n},\left.\mathbf{u}^{\prime}\right|_{n}\right)+\rho\left(\left.\mathbf{u}^{\prime}\right|_{n},\left.\mathbf{v}^{\prime}\right|_{n}\right)+\rho\left(\left.\mathbf{v}^{\prime}\right|_{n},\left.\mathbf{v}\right|_{n}\right)\right) \\
& =\rho\left(\mathbf{u}^{\prime}, \mathbf{v}^{\prime}\right),
\end{aligned}
$$

and similarly for the reverse inequality. We see that $\rho(\mathbf{u}, \mathbf{v})=\rho\left(\mathbf{u}^{\prime}, \mathbf{v}^{\prime}\right)$. Therefore we can define $\bar{\rho}$ on $\Sigma^{\infty} / \sim$ by $\bar{\rho}([\mathbf{u}],[\mathbf{v}])=\rho(\mathbf{u}, \mathbf{v})$. It is clear that this $\bar{\rho}$ on $\Sigma^{\infty} / \sim$ satisfies the triangle inequality; for $\bar{\rho}([\mathbf{u}],[\mathbf{v}])=0$, the first inequality of (4.5) in Theorem 4.2 implies that $\beta(\mathbf{u}, \mathbf{v})=+\infty$, which yields $[\mathbf{u}]=[\mathbf{v}]$. Therefore $\bar{\rho}$ is a metric on $\Sigma^{\infty} / \sim($ we will still use $\rho$ instead of $\bar{\rho}$ ).

Let $\psi: \Sigma^{\infty} / \sim \longrightarrow \mathcal{M}$ be defined by $\psi([\mathbf{u}])=\llbracket\left\{\left.\mathbf{u}\right|_{n}\right\} \rrbracket$. Then by Lemma 4.1, we have for any $\mathbf{u}, \mathbf{v} \in \Sigma^{\infty}$ that

$$
\rho(\psi([\mathbf{u}]), \psi([\mathbf{v})])=\rho\left(\llbracket\left\{\left.\mathbf{u}\right|_{n}\right\} \rrbracket, \llbracket\left\{\left.\mathbf{u}\right|_{n}\right\} \rrbracket\right)=\lim _{n \rightarrow \infty} \rho\left(\left.\mathbf{u}\right|_{n},\left.\mathbf{v}\right|_{n}\right)=\rho([\mathbf{u}],[\mathbf{v}]) .
$$


It follows that $\psi$ is an isometry. To show that $\psi$ is surjective, we let $\left\{\mathbf{w}_{n}\right\}$ be a $\rho$-Cauchy sequence in $\Sigma^{*}$. Since $\Sigma$ is a finite set, there exists a subsequence $\left\{\mathbf{w}_{n}^{(1)}\right\}$ such that the first letter of each term is $w_{1} \in \Sigma$. Inductively, we can extract a subsequence $\left\{\mathbf{w}_{n}^{(j+1)}\right\}$ of $\left\{\mathbf{w}_{n}^{(j)}\right\}$ such that the $(j+1)$-th letter of each term is $w_{j+1} \in \Sigma$. Note that $\left\{\mathbf{w}_{n}^{(n)}\right\}$ is a subsequence of $\left\{\mathbf{w}_{n}\right\}$. Hence $\left\{\mathbf{w}_{n}^{(n)}\right\}$ is also a $\rho$-Cauchy sequence in $\Sigma^{*}$, and $\left\{\mathbf{w}_{n}^{(n)}\right\} \sim\left\{\mathbf{w}_{n}\right\}$. Let $\mathbf{w}=w_{1} w_{2} \cdots w_{n} \cdots \in \Sigma^{\infty}$, then $\left.\mathbf{w}\right|_{n}=\left.\mathbf{w}^{(n)}\right|_{n}=w_{1} w_{2} \cdots w_{n}, n \geq 1$. Proposition 3.6 implies that $k\left(\mathbf{v}, \mathbf{w}_{n}^{(n)}\right)-$ $k\left(\mathbf{v},\left.\mathbf{w}\right|_{n}\right) \rightarrow 0$ as $n \rightarrow \infty$ for any $\mathbf{v} \in \Sigma^{*}$. Hence $\left\{\left.\mathbf{w}\right|_{n}\right\} \sim\left\{\mathbf{w}_{n}^{(n)}\right\} \sim\left\{\mathbf{w}_{n}\right\}$. i.e., $\psi([\mathbf{w}])=\llbracket\left\{\mathbf{w}_{n}\right\} \rrbracket$. This completes the proof.

We now identify the simple p.c.f. set with the Martin boundary.

Theorem 4.4. Suppose $K$ is a simple p.c.f. set, and let $\tilde{\pi}$ be the equivalence relation defined by the projection $\pi: \Sigma^{\infty} \rightarrow K$ as in (2.3). Then $K$ is homeomorphic to $\mathcal{M}$.

Proof. Let $\gamma=1-N^{-2}$ as in Theorem 4.2, and let $\Sigma^{\infty}$ be equipped with the metric $d(\mathbf{u}, \mathbf{v})=\gamma^{\alpha(\mathbf{u}, \mathbf{v})}$ where $\alpha(\mathbf{u}, \mathbf{v})=\max \left\{k:\left.\mathbf{u}\right|_{k}=\left.\mathbf{v}\right|_{k}\right\}$. It is well known that $\pi:\left(\Sigma^{\infty}, d\right) \rightarrow K$ is a continuous surjection and the quotient space $\Sigma^{\infty} / \pi$ is homeomorphic to $K$. On the other hand note that $\alpha(\mathbf{u}, \mathbf{v}) \leq \beta(\mathbf{u}, \mathbf{v})$. It follows from the second inequality of 4.5 in Theorem 4.2 that

$$
\rho(\mathbf{u}, \mathbf{v}) \leq C_{2} \gamma^{\beta(\mathbf{u}, \mathbf{v})} \leq C_{2} \gamma^{\alpha(\mathbf{u}, \mathbf{v})}=C_{2} d(\mathbf{u}, \mathbf{v}) .
$$

Hence the identity map $\iota:\left(\Sigma^{\infty}, d\right) \rightarrow\left(\Sigma^{\infty}, \rho\right)$ is continuous. It follows that $\iota$ : $\Sigma^{\infty} / \pi \rightarrow \Sigma / \approx$ is a continuous bijection. By compactness, they are homeomorphic, and by Theorem 4.2, $K$ and $\mathcal{M}$ are homeomorphic.

\section{Proof of Theorem 4.2}

We need a few technical lemmas to prove the upper and lower bounds of the Martin metric $\rho$ in Theorem 4.2. For a word $\mathbf{u} w_{0}, \mathbf{u} \in \Sigma^{*}$, we define the neighborhood of $\mathbf{u} w_{0}$ as

$$
\mathcal{N}\left(\mathbf{u} w_{0}\right)=\left\{\mathbf{v}: \mathbf{v} \in[\mathbf{u} a], a b \sim w_{0} d \text { for some } a, b, d \in \Sigma\right\} .
$$

(In the setting of a p.c.f. set, the last condition is just $K_{a} \cap K_{w_{0}} \neq \emptyset$ ). It is clear that $\left[\mathbf{u} w_{0}\right] \subseteq \mathcal{N}\left(\mathbf{u} w_{0}\right)$. We first give an estimate of the ancestor set and the neighborhood.

Lemma 5.1. For $\mathbf{w}_{n}=\mathbf{u} w_{0} w_{1} \cdots w_{n}, n \geq 1$, we have $\bigcup_{\mathbf{v} \in\left[\mathbf{w}_{n}\right]} \mathcal{A}_{n}(\mathbf{v}) \subseteq \mathcal{N}\left(\mathbf{u} w_{0}\right)$.

Proof. Let $\widetilde{\mathcal{A}}_{n}\left(\mathbf{w}_{n}\right)=\bigcup_{\mathbf{v} \in\left[\mathbf{w}_{n}\right]} \mathcal{A}_{n}(\mathbf{v})$. For $n=1$, let $\mathbf{v} \in\left[\mathbf{u} w_{0} w_{1}\right]$. If $w_{0}=w_{1}$, then $\mathcal{A}_{1}(\mathbf{v})=\left[\mathbf{u} w_{0}\right]$; otherwise, $\mathbf{v}=\mathbf{u} a b$ for some $a, b \in \Sigma$ with $a b \sim w_{0} w_{1}$ and $\mathcal{A}_{1}(\mathbf{v})=[\mathbf{u} a] \subseteq \mathcal{N}\left(\mathbf{u} w_{0}\right)$.

Assume the statement is true for $n$. Then

$$
\widetilde{\mathcal{A}}_{n+1}\left(\mathbf{w}_{n+1}\right)=\bigcup\left\{\mathcal{A}_{1}(\mathbf{v}): \mathbf{v} \in \widetilde{\mathcal{A}}_{n}\left(\mathbf{w}_{n+1}\right)\right\} \subseteq \bigcup\left\{\mathcal{A}_{1}(\mathbf{v}): \mathbf{v} \in \mathcal{N}\left(\mathbf{u} w_{0} w_{1}\right)\right\} .
$$

By repeating the above argument, we can show that for such $\mathbf{v}, \mathcal{A}_{1}(\mathbf{v}) \subseteq \mathcal{N}\left(\mathbf{u} w_{0}\right)$. Hence $\widetilde{\mathcal{A}}_{n+1}\left(\mathbf{w}_{n+1}\right) \subseteq \mathcal{N}\left(\mathbf{u} w_{0}\right)$. 
Lemma 5.2. For $\mathbf{w}_{n}=\mathbf{u} w_{0} w_{1} \cdots w_{n}, n \geq 1$, let $\mathbf{u} a \in \mathcal{A}_{n}\left(\mathbf{w}_{n}\right)$ with $a \neq w_{0}$. Then for $\mathbf{v} \in[\mathbf{u} a] \backslash\{\mathbf{u} a\}$,

$$
\mathbf{v} i \nsim \mathbf{u} w_{0} b, \quad \forall i, b \in \Sigma .
$$

Proof. We assume that $\#[\mathbf{u} a]>1$ (otherwise it is trivial), and write $\mathbf{u} a$ as $\mathbf{u} a=$ $\boldsymbol{\tau} c a^{k}$ where $k \geq 1$ and $c \neq a$. For $\mathbf{v} \in[\mathbf{u} a] \backslash\{\mathbf{u} a\}$, then $\mathbf{v}=\boldsymbol{\tau} e f^{k}$, where ef $\sim c a$ and $e \neq c$. There are two cases to consider:

Case $1(k>1)$. Comparing $\mathbf{u} w_{0} b=\boldsymbol{\tau} c a^{k-1} w_{0} b$ and $\mathbf{v} i=\boldsymbol{\tau} e f^{k} i$, and noting that $a \neq w_{0}$ and $c \neq e$, we see that $\mathbf{v} i \neq \mathbf{u} w_{0} b$ for any $i, b \in \Sigma$.

Case $2(k=1)$. Compare $\mathbf{u} w_{0} b=\tau c w_{0} b$ and $\mathbf{v} i=\tau$ efi. If $\mathbf{v} i \sim \mathbf{u} w_{0} b$, then $c w_{0} b \sim e f i$. This holds if and only if $w_{0}=b, f=i$ and $c w_{0} \sim e f$ (by (2.5)). Since ef $\sim c a,(\mathrm{P} 3)$ implies that $a=w_{0}$, a contradiction.

Lemma 5.3. Let $\mathbf{w}_{n}=\mathbf{u} w_{0} w_{1} \cdots w_{n}$. Then we have

$$
p_{n}\left(\mathbf{u} w_{0}, \mathbf{w}_{n}\right) \geq N^{-1} p_{n}\left(\boldsymbol{\tau}, \mathbf{w}_{n}\right) \quad \forall \boldsymbol{\tau} \in \mathcal{A}_{n}\left(\mathbf{w}_{n}\right) .
$$

Consequently, $p_{n}\left(\mathbf{u} w_{0}, \mathbf{w}_{n}\right) \geq N^{-(n+3)}$.

Proof. The inequality is trivial when $n=1$. Assume it is true for $n$. Let $\tau \in$ $\mathcal{A}_{n+1}\left(\mathbf{w}_{n+1}\right)$, then $\boldsymbol{\tau} \in \mathcal{N}\left(\mathbf{u} w_{0}\right)$ (by Lemma 5.1). If $\boldsymbol{\tau} \in\left[\mathbf{u} w_{0}\right]$, then we have $p_{n+1}\left(\boldsymbol{\tau}, \mathbf{w}_{n+1}\right)=p_{n+1}\left(\mathbf{u} w_{0}, \mathbf{w}_{n+1}\right)$ (by Proposition 3.2(ii)). Hence we need only prove the case that

$$
\boldsymbol{\tau} \in[\mathbf{u} a] \subset \mathcal{A}_{n+1}\left(\mathbf{w}_{n+1}\right), \quad \text { where } \quad a \neq w_{0} .
$$

Using Proposition 3.2(ii) again, we can assume that $\boldsymbol{\tau}=\mathbf{u} a$. Hence

$$
p_{n+1}\left(\boldsymbol{\tau}, \mathbf{w}_{n+1}\right)=p_{n+1}\left(\mathbf{u} a, \mathbf{w}_{n+1}\right)=\frac{1}{\sharp[\mathbf{u} a] N} \sum_{\mathbf{v}} p_{n}\left(\mathbf{v}, \mathbf{w}_{n+1}\right),
$$

where the sum is taken over for all $\mathbf{v} \in \mathcal{A}_{n}\left(\mathbf{w}_{n+1}\right)$ such that $\mathbf{v}^{-} \sim \mathbf{u} a$. It follows that $\mathbf{v} \in \mathcal{N}\left(\mathbf{u} w_{0} w_{1}\right) \subseteq \bigcup_{b \in \Sigma}\left[\mathbf{u} w_{0} b\right]$. But by Lemma 5.2 we have $\mathbf{v}^{-}=\mathbf{u} a$. Note that $a \neq w_{0}$, we get only one pair $(c, b)$ such that $\mathbf{v}=\mathbf{u} a c \sim \mathbf{u} w_{0} b$. Hence, there is only one term in the sum. Then we have

$$
\begin{aligned}
p_{n+1}\left(\boldsymbol{\tau}, \mathbf{w}_{n+1}\right) & =\frac{1}{\#[\mathbf{u} a] N} \cdot p_{n}\left(\mathbf{u} a c, \mathbf{w}_{n+1}\right) \\
& =\frac{1}{\#[\mathbf{u} a] N} \cdot p_{n}\left(\mathbf{u} w_{0} b, \mathbf{w}_{n+1}\right) \\
& =\frac{\#\left[\mathbf{u} w_{0}\right]}{\#[\mathbf{u} a]} p\left(\mathbf{u} w_{0}, \mathbf{u} w_{0} b\right) p_{n}\left(\mathbf{u} w_{0} b, \mathbf{w}_{n+1}\right) \\
& \leq N p_{n+1}\left(\mathbf{u} w_{0}, \mathbf{w}_{n+1}\right) .
\end{aligned}
$$

This proves the first part. For the second part, making use of Corollary 3.3 and the above, we have

$$
1=N^{n} \sum_{\boldsymbol{\tau} \in \mathcal{A}_{n}\left(\mathbf{w}_{n}\right)} p_{n}\left(\boldsymbol{\tau}, \mathbf{w}_{n}\right) \leq N^{n+1} p_{n}\left(\mathbf{u} w_{0}, \mathbf{w}_{n}\right) \# \mathcal{A}_{n}\left(\mathbf{w}_{n}\right) .
$$

Then by Lemma5.1 and (P3), we have $\mathcal{A}_{n}\left(\mathbf{w}_{n}\right) \leq N^{2}$ and the assertion follows. 
Proof of Theorem 4.2. Let $\mathbf{u}, \mathbf{v} \in \Sigma^{\infty}$ such that $\mathbf{u} \nsim \mathbf{v}$. Let $m=\beta(\mathbf{u}, \mathbf{v})=$ $\max \left\{k:\left.\left.\mathbf{u}\right|_{k} \sim \mathbf{v}\right|_{k}\right\}$. Then $\mathbf{u}$ and $\mathbf{v}$ have representations

$$
\mathbf{u}=\boldsymbol{\tau} x_{1} x_{2} \cdots \quad \text { and } \quad \mathbf{v}=\boldsymbol{\tau}^{\prime} y_{1} y_{2} \cdots,
$$

where $\boldsymbol{\tau} \sim \boldsymbol{\tau}^{\prime}$ and $|\boldsymbol{\tau}|=\left|\boldsymbol{\tau}^{\prime}\right|=m$. Note that $\boldsymbol{\tau}=\left.\mathbf{u}\right|_{m}$ and $\boldsymbol{\tau}^{\prime}=\left.\mathbf{v}\right|_{m}$.

The upper bound estimate. By Proposition 3.2 (iii), we have that $k\left(\mathbf{w},\left.\mathbf{u}\right|_{m+1}\right)=$ $k\left(\mathbf{w},\left.\mathbf{v}\right|_{m+1}\right)$ for any $\mathbf{w}$ with $|\mathbf{w}| \leq m$. Hence for $|\mathbf{w}| \leq m \leq n$, by Proposition 3.6. we have the estimation

$$
\begin{aligned}
& \left|k\left(\mathbf{w},\left.\mathbf{u}\right|_{n}\right)-k\left(\mathbf{w},\left.\mathbf{v}\right|_{n}\right)\right| \leq\left|k\left(\mathbf{w},\left.\mathbf{u}\right|_{n}\right)-k\left(\mathbf{w},\left.\mathbf{u}\right|_{m+1}\right)\right| \\
+ & \left|k\left(\mathbf{w},\left.\mathbf{v}\right|_{n}\right)-k\left(\mathbf{w},\left.\mathbf{v}\right|_{m+1}\right)\right| \leq \frac{2 N^{|\mathbf{w}|} \gamma^{m-|\mathbf{w}|}}{1-\gamma}
\end{aligned}
$$

On the other hand for $m<|\mathbf{w}| \leq n$, by (4.1),

$$
\left|k\left(\mathbf{w},\left.\mathbf{u}\right|_{n}\right)-k\left(\mathbf{w},\left.\mathbf{v}\right|_{n}\right)\right| \leq 2 N^{|\mathbf{w}|} .
$$

It follows that

$$
\rho\left(\left.\mathbf{u}\right|_{n},\left.\mathbf{v}\right|_{n}\right) \leq \sum_{k=0}^{m}\left(\frac{r}{N}\right)^{k} \frac{2 N^{k} \gamma^{m-k}}{1-\gamma}+\sum_{k=m+1}^{n} 2\left(\frac{r}{N}\right)^{k} N^{k} \leq C_{2} \gamma^{m} .
$$

Let $n \rightarrow \infty$, we obtain the upper bound estimate.

The lower bound estimate. Denote by $\mathbf{w}=\left.\mathbf{u}\right|_{m+3}$. We claim that $k\left(\mathbf{w},\left.\mathbf{v}\right|_{n}\right)=0$ for $n>|\mathbf{w}|=m+3$. If otherwise, Lemma 5.1 implies that

$$
\mathbf{w} \in \mathcal{A}_{n-|\mathbf{w}|}\left(\left.\mathbf{v}\right|_{n}\right) \subset\left\{\mathbf{w}^{\prime}: \mathbf{w}^{\prime} \in\left[\left.\mathbf{v}\right|_{m+2} a\right], a \in \Sigma\right\} .
$$

Hence $\mathbf{w}=\left.\left.\mathbf{u}\right|_{m+3} \sim \mathbf{v}\right|_{m+2} a$ for some $a \in \Sigma$. It follows that either $\left.\mathbf{u}\right|_{m+1}=\left.\mathbf{v}\right|_{m+1}$ if $a$ is not equal to the last alphabet of $\left.\mathbf{v}\right|_{m+2}$, or $\left.\left.\mathbf{u}\right|_{m+2} \sim \mathbf{v}\right|_{m+2}$ otherwise. This contradicts that $\beta(\mathbf{u}, \mathbf{v})=m$. We complete the proof of the claim.

On the other hand, by Lemma 5.3 , we have $k\left(\mathbf{w},\left.\mathbf{u}\right|_{n}\right) \geq N^{|\mathbf{w}|-3}=N^{m}$. Now we have the following estimation:

$$
\begin{aligned}
\rho(\mathbf{u}, \mathbf{v}) & =\lim _{n \rightarrow \infty} \rho\left(\left.\mathbf{u}\right|_{n},\left.\mathbf{v}\right|_{n}\right) \\
& \geq \lim _{n \rightarrow \infty}\left(\frac{r}{N}\right)^{m+3}\left|k\left(\mathbf{w},\left.\mathbf{u}\right|_{n}\right)-k\left(\mathbf{w},\left.\mathbf{v}\right|_{n}\right)\right| \\
& \geq N^{m}\left(\frac{r}{N}\right)^{m+3}=C_{1} r^{m} .
\end{aligned}
$$

Remark. If we take $r=\gamma$ in the definition of Martin metric $\rho(\cdot, \cdot)$ in (4.2), then we can improve the estimation in Theorem 4.2 to

$$
C_{1} \gamma^{\beta(\mathbf{u}, \mathbf{v})} \leq \rho(\mathbf{u}, \mathbf{v}) \leq C_{2} \beta(\mathbf{u}, \mathbf{v}) \gamma^{\beta(\mathbf{u}, \mathbf{v})} .
$$

To see this, we need only observe that the term $\sum_{k=0}^{m}\left(\frac{r}{N}\right)^{k} N^{k} \gamma^{m-k}$ in (5.2) is bounded by a multiple of $\beta(\mathbf{u}, \mathbf{v}) \gamma^{\beta(\mathbf{u}, \mathbf{v})}$. 


\section{The SPACE OF EXITS}

For a function $h: \Sigma^{*} \rightarrow \mathbb{R}$, we let $P h(\mathbf{u})=\sum_{\mathbf{v} \in \Sigma^{*}} p(\mathbf{u}, \mathbf{v}) h(\mathbf{v})$, and call $h$ harmonic (with respect to $P$ ) if $P h(\mathbf{u})=h(\mathbf{u})$ for all $\mathbf{u} \in \Sigma^{*}$. From the definition of Martin boundary, we can extend $k(\mathbf{v}, \mathbf{u}), \mathbf{v}, \mathbf{u} \in \Sigma^{*}$ to $k(\mathbf{v}, y), y \in \mathcal{M}$. It is well known that $k(\cdot, y)$ is harmonic for $y \in \mathcal{M}$, and for any nonnegative harmonic function $h$, there exists a measure $\mu_{h}$ (which may not be unique) such that

$$
h(\cdot)=\int_{\mathcal{M}} k(\cdot, y) d \mu_{h}(y) .
$$

The measure $\mu_{h}$ is called the spectral measure of $h$. For $y \in \mathcal{M}$, with a slight abuse of notation, we use the notation $k_{y}(\cdot)$ and $k(\cdot, y)$ interchangeably. Following Dynkin Dy, the set of all $y \in \mathcal{M}$, for which $\mu_{k_{y}}=\delta_{y}$ (the point mass measure at $y$ ), is called the space of exits, and is denoted by $\mathcal{M}_{\text {min. }}$. It is known that the spectral measure in (6.1) can be chosen to be supported in $\mathcal{M}_{\min }$ and is unique ([Dy, [P], W] $)$.

Theorem 6.1. Let $\mathcal{M}$ be the Martin boundary induced by the transition probability in (3.1). Then $\mathcal{M}_{\min }=\mathcal{M}$.

Proof. We claim that for any $\xi \in \mathcal{M}, \xi \neq y$, there exists $\mathbf{v} \in \Sigma^{*}$ such that $k(\mathbf{v}, y)=0$, but $k(\mathbf{v}, \xi)>0$. Indeed, we let $\beta(\xi, y)=\max \left\{k:\left.\left.\xi\right|_{k} \sim y\right|_{k}\right\}$ (as in (4.4)). Since $\xi \neq y$ in $\mathcal{M}$, Theorem 4.2 implies $\beta(\xi, y)=s<\infty$, i.e., $\left.\left.\xi\right|_{m} \not y\right|_{m}$ for all $m>s$. Let $\mathbf{v}=\left.\xi\right|_{s+3}$. As in the proof of Theorem 4.2 (the lower bound estimate), we have $k\left(\mathbf{v},\left.y\right|_{m}\right)=0$ for all $m>s+3$, so that $k(\mathbf{v}, y)=0$. On the other hand, it follows from Lemma 5.3 that $k(\mathbf{v}, \xi)=k\left(\left.\xi\right|_{s+3}, \xi\right)>0$, and the claim follows.

The claim implies that $\bigcup_{\mathbf{v} \in \Sigma^{*}: k(\mathbf{v}, y)=0}\{\xi: k(\mathbf{v}, \xi)>0\}=\mathcal{M} \backslash\{y\}$. Now for any $\mathbf{v} \in \Sigma^{*}$ such that $k(\mathbf{v}, y)=0$, 6.1) implies (taking $h(\cdot)=k_{y}(\cdot)$ ) that $\mu_{k_{y}}\{\xi: k(\mathbf{v}, \xi)>0\}=0$. Hence $\operatorname{supp}\left(\mu_{k_{y}}\right)$ can only be $\{y\}$. This completes the proof.

Next we consider the hitting distribution $\mu_{1}$ of the limit $X_{\infty}$ of the Markov chain $\left\{X_{n}\right\}_{n=0}^{\infty}$ (starting from the root $\emptyset$ ); it is also the spectral measure of the constant function 1 in (6.1) W]. We can identify $\mu_{1}$ more explicitly in the following proposition. For $\mathbf{w} \in \Sigma^{*}$ with $|\mathbf{w}|=\ell$, let $C(\mathbf{w})=\left\{\mathbf{u} \in \Sigma^{\infty}:\left.\mathbf{u}\right|_{\ell}=\mathbf{w}\right\}$ be the cylinder set for $\mathbf{w}$. Let $\nu$ be the Bernoulli measure on $\Sigma^{*}$ such that $\nu(C(\mathbf{w}))=$ $1 / N^{\ell}, \mathbf{w} \in \Sigma^{*}$. It is clear that from 2.5 that $\nu([\mathbf{u}])=0$ for each $\sim$ equivalent class $[\mathbf{u}]$ in $\Sigma^{\infty}$. Hence we can also regard $\nu$ as a measure on $\Sigma^{\infty} / \sim$.

Proposition 6.2. The hitting distribution $\mu_{1}$ equals the Bernoulli measure $\nu$ on $\mathcal{M}\left(=\Sigma^{\infty} / \sim\right)$.

Proof. In view of the fact that $\mu_{1}$ is uniquely determined by $\int_{\mathcal{M}} k(\mathbf{v}, \xi) d \mu_{1}(\xi)=1$ for all $\mathbf{v} \in \Sigma^{*}$ and the identifications on $\Sigma^{\infty}$ and $\Sigma^{\infty} / \sim$, we need only show that

$$
\int_{\Sigma^{\infty}} k(\mathbf{v}, \xi) d \nu(\xi)=1 \quad \forall \mathbf{v} \in \Sigma^{*}
$$


This follows from a straightforward calculation:

$$
\begin{aligned}
\int_{\Sigma^{\infty}} k(\mathbf{v}, \xi) d \nu(\xi) & =\int_{\Sigma^{\infty}} \lim _{n \rightarrow \infty} k\left(\mathbf{v},\left.\xi\right|_{n}\right) d \nu(\xi) \\
& =\lim _{n \rightarrow \infty} \int_{\Sigma^{\infty}} k\left(\mathbf{v},\left.\xi\right|_{n}\right) d \nu(\xi) \\
& =\lim _{n \rightarrow \infty} \sum_{\mathbf{w} \in \Sigma^{n}} k(\mathbf{v}, \mathbf{w}) \nu(C(\mathbf{w})) \\
& =\lim _{n \rightarrow \infty} \sum_{\mathbf{w} \in \Sigma^{n}} N^{n} p_{n-|\mathbf{v}|}(\mathbf{v}, \mathbf{w}) \cdot\left(1 / N^{n}\right)=1
\end{aligned}
$$

(the interchange of the limit follows from $k\left(\mathbf{v},\left.\xi\right|_{n}\right) \leq N^{|\mathbf{v}|}$ and the bounded convergence theorem).

Recall that for an IFS $\left\{S_{j}\right\}_{j=1}^{N}$ in $\mathbb{R}^{d}$, an important class of invariant measures on $K$ is defined by

$$
\mu(B)=\sum_{j=1}^{N} p_{j} \mu\left(S_{j}^{-1}(B)\right),
$$

where $\left\{p_{j}\right\}_{j=1}^{N}$ are probability weights and $B$ is any Borel subset in $\mathbb{R}^{d}[\underline{\mathrm{Hu}}$. It is easy to see that if the IFS satisfies the open set condition, then $\mu\left(K_{\mathbf{v}}\right)=p_{v_{1}} \cdots p_{v_{n}}$ for $\mathbf{v}=v_{1} \cdots v_{n}$.

Corollary 6.3. Suppose the simple p.c.f. set $K$ is defined by an IFS $\left\{S_{j}\right\}_{j=1}^{N}$ of similitudes with equal contraction (i.e., $S_{j}(x)=\rho A x+d_{j}$ where $0<\rho<1, A$ is an orthogonal matrix and $\left.d_{j} \in \mathbb{R}^{d}\right)$. Then the measure $\mu_{1}$ is the invariant measure with probability weights $p_{j}=1 / N$ for each $1 \leq j \leq N$. Moreover, it is an $\alpha$-Hausdorff measure on $K$ with $\alpha=\log N /|\log \rho|$.

Proof. As was proved in DL, such an IFS satisfies the open set condition. The statement follows readily from Proposition 6.2.

\section{ACKNOWLEDGEMENTS}

Part of this work was carried out while the second author was visiting the Université Bretagne-Sud and the third author was visiting the Chinese University of Hong Kong. They are grateful to the Departments of Mathematics for their hospitality and help during their visits. They also thank Professor S.M. Ngai for reading through the manuscript and for many valuable suggestions.

\section{REFERENCES}

[A] A. Ancona, Positive harmonic functions and hyperbolicity, Potential Theory. Surveys and Problems, (eds, J. Kral, et al.), Lecture Notes in Math., no. 1344, Springer, 1987, 128-136. MR 973878

[BR] C. Bandt and H. Rao, Topology and separation of self-similar fractals in the plane, Nonlinearity, 20 (2007), 1463-1474. MR2327133 (2008g:28036)

[D] J. Doob, Discrete potential theory and boundaries, J. Math. and Mech., 8 (1959), 433-458. MR0107098(21:5825)

[DIK] M. Denker, A. Imai and S. Koch, Dirichlet forms on quotients of shift spaces, Colloq. Math., 107 (2007), 57-80. MR 2283132 (2008b:28010)

[DL] Q. Deng and K. S. Lau, Open set condition and post-critically finite self-similar sets, Nonlinearity, 21 (2008), 1227-1232. MR2422376 (2009j:28020) 
[DS1] M. Denker and H. Sato, Sierpiński gasket as a Martin boundary I: Martin kernel, Potential Anal., 14 (2001), 211-232. MR1822915 (2002f:60139)

[DS2] M. Denker and H. Sato, Sierpiński gasket as a Martin boundary II: The intrinsic metric, Publ. RIMS, Kyoto Univ., 35 (1999), 769-794. MR1739300(2002f:60140)

[DS3] M. Denker and H. Sato, Reflections on harmonic analysis of the Sierpiński gasket, Math. Nachr. 241 (2002), 32-55. MR1912376 (2003e:28016)

[Dy] E. Dynkin, Boundary theory of Markov processes (the discrete case), Russian Math. Surveys, 24 (1969), 1-42. MR0245096 (39:6408)

[F] K. Falconer, Fractal geometry, Mathematical Foundations and Applications, Wiley, 1990. MR:1102677 (92j:28008)

[H] G. Hunt, Markov chains and Martin boundaries, Illinois J. Math., 4 (1960), 313-340. MR0123364 (23:A691)

[Hu] J. Hutchinson, Fractal and self-similarity, Indiana Univ. Math. J., 30 (1981), 712-747. MR625600 (82h:49026)

[I] A. Imai, The difference between letters and a Martin kernel of a modulo 5 Markov chain, Adv. in Appl. Math. 28 (2002), no. 1, 82-106. MR.1884389(2003f:60126)

[K1] J. Kigami, Analysis on Fractals, Cambridge University Press, 2001. MR 1840042 (2002c:28015)

[K2] J. Kigami, Harmonic calculus on p.c.f. self-similar sets, Trans. Amer. Math. Soc., 335 (1993), 721-755. MR 1076617(93d:39008)

[Ka] V. Kaimanovich, Random walks on Sierpinssi graphs: hyperbolicity and stochastic homogenization, Fractals in Graz 2001, 145-183, Trends Math., Birkhäuser, 2003. MR2091703 (2005h:28022)

[LW] K. S. Lau and X. Y. Wang, Self-similar sets as hyperbolic boundary, Indiana Univ. Math. J., 58 (2009), 1777-1795. MR2542979

[M] R. Martin, Minimal positive harmonic functions, Trans. Amer. Math. Soc., 49 (1941), 137-172. MR0003919(2:292h)

[P] R. Phelps, Lectures on Choquet's theorem, 2rd ed., Lectures Notes in Math., no. 1757, Springer, 2001. MR.1835574 (2002k:46001)

[S1] R. Strichartz, Differential equations on fractals: a tutorial, Princeton University Press, 2006. MR2246975 (2007f:35003)

[S2] R. Strichartz, The Laplacian on the Sierpiniski gasket via the method of averages, Pacific J. Math., 201 (2001), 241-256. MR.1867899(2003f:35056)

[S3] R. Strichartz, Analysis on product of fractals, Trans. Amer. Math. Soc., 355 (2003), 40194043. MR1990573 (2004b:28013)

[W] W. Woess, Random walks on infinite graphs and groups, Cambridge University Press, 2000. MR:1743100(2001k:60006)

Department of Mathematics, Chinese University of Hong Kong, Shatin, Hong Kong

Department of Mathematics, Chinese University of Hong Kong, Shatin, Hong Kong

E-mail address: kslau@math.cuhk.edu.hk

School of Mathematics and Computational Science, Sun Yat-Sen University, GuangZhou 510275, People's Republic of China

E-mail address: mcswxy@mail.sysu.edu.cn 